\title{
Mechanisms of action of insecticides on ligand-gated ion channels*
}

\author{
Makoto IHARA** \\ School of Agriculture, Kinki University, 3327-204 Naka-machi, Nara 631-8505, Japan
}

(Accepted May 20, 2007)

\begin{abstract}
Ligand-gated ion channels (LGICs) mediate fast synaptic neurotransmission and are important targets for insecticides. Thus, the actions of several insecticides have been explored in electrophysiological studies on recombinant and native insect neuronal LGICs. I have shown that non-competitive antagonists of $\gamma$-aminobutyric acid gated $\mathrm{Cl}^{-}$channels also act on glutamate-gated $\mathrm{Cl}^{-}$channels, albeit at higher concentrations. Neonicotinoids are more potent agonists on recombinant hybrid nicotinic acetylcholine receptors (nAChRs) consisting of Drosophila $\mathrm{D} \alpha 2$ and vertebrate $\beta 2$ subunits than those consisting of only vertebrate nAChR subunits $(\alpha 4 \beta 2)$. Using this hybrid $\mathrm{nAChR}$, clothianidin and related compounds containing a acyclic guanidine moiety were found to be super-agonists. Similar super-agonist actions of neonicotinoids were also observed on cultured Drosophila cholinergic neurons. Single channel nAChR recordings show that a clothianidin analogue induces a high conductance state in channel opening more frequently than acetylcholine, thereby offering a possible explanation for its super-agonist action. Unlike the case for clothianidin, imidacloprid attenuates the acetylcholine-induced response of native neuronal nAChRs when co-applied with ACh. These new discoveries add to our understanding of both the selectivity and the diverse actions of insecticides targeting LGICs. (C) Pesticide Science Society of Japan
\end{abstract}

Keywords: ligand-gated ion channels (LGIC), electrophysiology, neurotoxic insecticides.

\section{Introduction}

Insect nervous system is a target of many classes of insecticides. Voltage-sensitive sodium channels, $\gamma$-aminobutyric acid gated $\mathrm{Cl}^{-}$channels (GABACls) and nicotinic acetylcholine receptors $(\mathrm{nAChR})$ are the major target of insecticides. In addition to these, glutamate-gated chloride channels (GluCls) which are anion-permeable channels identified only in invertebrate. Of these insecticide targets, nAChRs, $\mathrm{GABACl}$ and $\mathrm{GluCls}$ belong to the cys-loop super family of ligand-gated ion channels (LGICs). The members of cys-loop superfamily share similar structural features: they consist of five subunit proteins which possess four transmembrane helices and large N-terminus ligand binding domain.

Neonicotinoids have been shown to act as agonists or partial agonists on $\mathrm{nAChR}$. However, Nishimura et al. reported

\footnotetext{
* See Part II for the full Japanese article.

** To whom correspondence should be addressed. Present address: RIKEN Harima Institute, SPring-8 Center, 1-1-1 Kouto, Sayo-chou, Sayo-gun, Hyogo 679-5148, Japan E-mail: makoto_i@spring8.or.jp

(C) Pesticide Science Society of Japan
}

that neonicotinoids showed nerve blocking action followed by excitation, suggesting the actions are not limited to agonists. On the other hand, non-competitive antagonists of GABACl, such as fipronil and dieldrin, have been reported to act on GluCls as well as GABACls. However, little is known about the selectivity for GABACls over GluCls of non-competitive LGIC antagonists. Thus the author has employed voltageclamp electrophysiology to elucidate the mechanism of action and selectivity of insecticides on native LGICs expressed in American cockroach neuron and recombinant LGICs expressed in Xenopus laevis oocytes.

\section{Establishing a whole-cell patch clamp electrophysiology that permits stable recording of responses to agonists of LGICs expressed in American cockroach neurons}

Binding assay using radioactive ligands is useful for high throughput evaluation of affinity for LGICs of compounds as well as studying their mode of actions by displacement kinetics. Yet it is difficult with this method to analyze agonist/antagonist actions on the channels. Measuring radioactive ion influx through membrane fractions is a way to evaluate agonist actions on LGIC, but it is impossible with this method to 
analyze detailed channel gating kinetics of LGICs. In this respect voltage-clamp electrophysiology is superior because it permits realtime analysis of antagonist as well as agonist actions on the receptor channels.

Antagonist actions of hydrophobic insecticides often take time to reach a steady state. It is therefore essential to establish a patch clamp method that permits stable recording of LGIC responses. However, a marked rundown of GABA and glutamate induced responses of the terminal abdominal ganglion (TAG) neurons occurred when intracellular solution with no additives was used to record the response. By investing several additives to resolve this problem, addition of sodium pyruvate at a certain concentration to the pipette solution prevent the rundown, permitting stable recordings of the GABA and glutamate-induced responses of the cockroach neurons for longer than $1 \mathrm{hr}$. Under this condition, it was found that both GABA and glutamate-induced responses of the cockroach neuron are mediated by chloride ion and GABA and glutamate showed similar dose-response relationships.

\section{Selectivity of GABACI ligand on native and recombinant GABA- and glutamate-gated chloride channels}

4'-Ethynyl-4- $n$-propylbicycloorthobenzoate (EBOB) has been employed intensively as a radioligand in binding assays to evaluate the pharmacology of GABACls of insects and mammals, whereas $\gamma$-hexachlorocyclohexane $(\gamma$-HCH) was earlier used as an insecticide that targets insect GABACls. Since recent studies have shown that not only GABACls but also glutamate-gated chloride channels (GluCls) of cockroach thoracic ganglion neurons are blocked by picrotoxinin, dieldrin and fipronil, the actions of $\mathrm{EBOB}$ and $\gamma-\mathrm{HCH}$ on native GABACl and GluCls on cockroach TAG neurons were evaluated. EBOB and $\gamma-\mathrm{HCH}$ were found to block not only the GABA- but also glutamate-gated responses, with the actions augmented by repeated co-application with agonists. It was also found that prolonged pre-application of EBOB and $\gamma$ $\mathrm{HCH}$ prior to co-application with GABA and glutamate resulted in enhanced blocking actions, indicating resting-state modulation of the channels. The blocking actions of EBOB and $\gamma-\mathrm{HCH}$ on the GABA- and glutamate-induced responses were compared by determining $\mathrm{IC}_{50}$ values when the actions reached a steady state. As a consequence, both EBOB and $\gamma$ $\mathrm{HCH}$ were found to act selectively on the $\mathrm{GABACl}$ of the cockroach TAG neuron.

Furthermore, actions of fipronil, $\gamma-\mathrm{HCH}$ and picrotoxinin on recombinant house fly GABACl (MdRDL) and $\mathrm{GluCl}$ (MdGluCl) expressed in Xenopus laevis oocytes were evaluated to clarify if the selectivity of insecticides to GABACl over $\mathrm{GluCl}$ observed in the cockroach TAG neurons were also observed for other insect receptors. Two-electrode voltage clamp electrophysiology was employed to investigate the actions of compounds on recombinant MdRDL and MdGluCl which were expressed in Xenopus oocytes by injecting cRNAs. All the compounds tested showed blocking actions on not only MdRDL but also MdGluCl, and the order of selectivity was evaluated by their $\mathrm{pIC}_{50}\left(-\log \mathrm{IC}_{50}\right)$ values. The order of the selectivity to MdRDL over MdGluCl was picrotoxinin $>\gamma$-HCH $>$ fipronil, suggesting that fipronil action on the $\mathrm{MdGluCl}$ as well as on $\mathrm{MdGluCl}$ is likely contribute to its toxicity. In addition, the Hill coefficient for its blocking action on the MdGluCl was significantly smaller than that for its action on MdRDL, suggesting a different mode of action of fipronil between MdRDL and MdGluCl. In addition, the author found that co-expression of MdGluCl with MdRDL in Xenopus oocytes significantly enhanced the amplitude of the GABA-evoked response but did not affect agonist, modulator and antagonist pharmacology of the two channels.

\section{Diverse actions of neonicotinoids on native and recombinant $\mathrm{nAChRs}$}

To date, genomics studies have identified many nAChR subunit genes together with their post transcriptional RNA editing and alternative splicing. In general $\mathrm{nAChRs}$ are heteropentamers, so many $\mathrm{nAChR}$ subtypes presumably present in native tissues. To facilitate understandings the pharmacology of neonicotinoids, actions of recombinant nAChRs expressed in Xenopus oocytes were evaluated. It was found that neonicotinoids acted more strongly on hybrid $\mathrm{nAChR}$ consisting of Drosophila $\mathrm{D} \alpha 2$ and vertebrate $\beta$ subunits than vertebrate $\mathrm{nAChRs}$, suggesting that the $\mathrm{D} \alpha 2$ subunit possesses the structural features favorable for interaction with neonicotinoids. Although imidacloprid was a very weak agonist on the $\alpha 4 \beta 2 \mathrm{nAChR}$, it potentiated the acetylcholine induced responses when co-applied with ACh at concentrations at which it did not appear to evoke any currents.

When tested on the $\mathrm{D} \alpha 2 \beta 2 \mathrm{nAChR}$, clothianidin evoked larger responses than ACh. Structure activity studies showed that the acyclic guanidine moiety of clothianidin is involved in such a super agonist action. It was also found that the affinity for the hybrid nAChRs is increased by replacing the nitroimine moiety by nitromethylene moiety. The underlying mechanism was studied by calculating interaction energy for imidacloprid and its nitromethylene analog as complex formed with hydrogen bondable model molecules. It was found that the nitromethylene unit can more stabilize the complex with the model molecules than the nitroimine unit.

Structure-agonist action relationship for neonicotinoids on nAChRs expressed by native insect neurons was studied using patch clamp electrophysiology. Neonicotinoids acted on the cockroach neurons as partial agonist, however the structure activity relationship observed resembled that observed in the D $\alpha 2 \beta 2$ nAChRs. Namely, neonicotinoids with an acyclic structure at the nitro guanidine or related moiety evoked greater responses than the neonicotinoids with ring structures. In addition, nitromethylene neonicotinoids showed higher agonist potency in terms of $\mathrm{EC}_{50}$ values compared to com- 
pounds with a nitroimine moiety.

The agonist potency of neonicotinoids was compared with their insecticidal activity against American cockroaches. It was found that not only agonist potency but also antagonist action at very low concentrations and hydrophobicity of compounds are implicated in the insecticidal activity.

Even though clothianidin was a partial agonist of the cockroach TAG neuron, it showed a super agonist action on Drosophila cholinergic neurons expressing the D $\alpha 2$ subunit. Single channel analysis showed that clothianidin analogue P$\mathrm{CH}-\mathrm{CTD}$ enhances opening of $\mathrm{nAChR}$ channels at the highest conductance state more frequently than $\mathrm{ACh}$, accounting for its super agonist action.

\section{Elucidation of the mode of binding of neonicotinoids on $\mathrm{nAChR}$}

As mentioned above, neonicotinoids show very diverse action on nAChRs, and they are selective on insect nAChRs, which are the result of physicochemical and steric interactions between neonicotinoids and their binding sites in nAChRs. The author has for the first time predicted the formation and breakage of hydrogen bond (HB) networks likely plays a key role in the diversity and selectivity. In the model, the author has demonstrated that formation and breakage of $\mathrm{HB}$ at the nitro group with agonist binding loops and at the position 1 $\mathrm{NH}$ with the backbone carbonyl in loop B can influence the efficacy as well as the affinity of neonicotinoids.

All these results are believed to enhance our understanding of mode of actions and mechanism for the selectivity of insecticides and therefore will contribute to the development of novel and safer insecticides that target insect LGICs. 